\title{
KirigamiTable: Designing for Proxemic Transitions with a Shape-Changing Tabletop
}

\author{
Jens Emil Grønbæk ${ }^{1}$, Majken Kirkegaard Rasmussen², Kim Halskov³, \\ Marianne Graves Petersen ${ }^{1}$ \\ ${ }^{1}$ Department of Computer Science, Aarhus University, Aarhus, Denmark \\ ${ }^{2}$ Department of Engineering, Aarhus University, Aarhus, Denmark \\ ${ }^{3}$ CAVI, Aarhus University, Aarhus, Denmark \\ jensemil@cs.au.dk, mkirkegaard@eng.au.dk, halskov@cavi.au.dk, mgraves@cs.au.dk
}
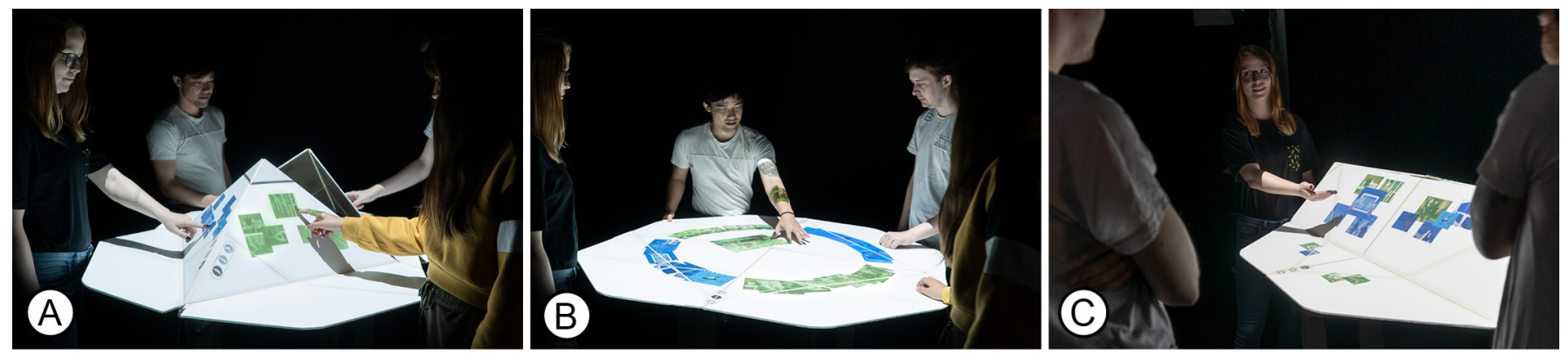

Figure 1. KirigamiTable is a shape-changing tabletop, supporting proxemic transitions in collaborative work. It deploys a kirigami pattern that allows for transformation between three shapes - four-fold (A), flat-fold (B) and two-fold (C) - for working individually or together on a single tabletop.

\section{ABSTRACT}

A core challenge in tabletop research is to support transitions between individual activities and team work. Shape-changing tabletops open up new opportunities for addressing this challenge. However, interaction design for shape-changing furniture is in its early stages - so far, research has mainly focused on triggering shape-changes, and less on the actual interface transitions. We present KirigamiTable - a novel actuated shape-changing tabletop for supporting transitions in collaborative work. Our work builds on the concept of Proxemic Transitions, considering the dynamic interplay between social interactions, interactive technologies and furniture. With KirigamiTable, we demonstrate the potential of interactions for proxemic transitions that combine transformation of shape and digital contents. We highlight challenges for shape-changing tabletops: initiating shape and content transformations, cooperative control, and anticipating shape-change. To address these challenges, we propose a set of novel interaction techniques, including shape-first and content-first interaction, cooperative gestures, and physical and digital preview of shape-changes.

Permission to make digital or hard copies of all or part of this work for personal or classroom use is granted without fee provided that copies are not made or distributed for profit or commercial advantage and that copies bear this notice and the full citation on the first page. Copyrights for components of this work owned by others than ACM must be honored. Abstracting with credit is permitted. To copy otherwise, or republish, to post on servers or to redistribute to lists, requires prior specific permission and/or a fee. Request permissions from permissions@acm.org.

CHI '20, April 25-30, 2020, Honolulu, HI, USA.

(C) 2020 Association for Computing Machinery.

ACM ISBN 978-1-4503-6708-0/20/04 ...\$15.00.

http://dx.doi.org/10.1145/3313831.3376834

\section{Author Keywords}

Shape-changing interfaces; interactive tabletops; proxemics; collaboration; transitions; interaction techniques

\section{CCS Concepts \\ -Human-centered computing $\rightarrow$ Interaction design;}

\section{INTRODUCTION}

Interactive tabletops have shown great promise for supporting collaboration [59, 63, 27]. However, several researchers have pointed out that a core challenge for research on shared displays (such as tabletops and wall-displays) is to support transitions between different ways of engaging with co-located others $[16,64,6,19,50]$. Recent research in actuated shape displays has begun to address this challenge through design of shape-changing furniture [13, 42, 29, 66, 67, 19]. With TRANSFORM, Leithinger et al. [42] demonstrate how a shape-changing tabletop can support a variety of activities. Grønbæk et al. [19] coin the term Proxemic Transitions, considering how transitions in people's social interactions are conditioned by the interplay between interactive technologies and features in the environment such as furniture. They illustrate through a prototype how shape-changing furniture enable people to re-organize themselves with respect to interpersonal distance and orientation as well as in relation to displays and other artefacts. Takashima et al. [66] explore how the shape of a tabletop display can influence users' spatial arrangements and the atmosphere around it, and Takashima et al. [67] explore the properties of different vertical display shapes, motivating further research on shape-changing display 
transitions. These examples are early experiments, and a number of challenges await further exploration. This includes the challenges of constructing such interfaces, designing ways of interacting with them, and exploring their design more specifically within the context of collaboration practices.

Inspired by prior research, we propose a set of interaction techniques to address challenges of designing for proxemic transitions around a shape-changing tabletop. We developed these through the design and implementation of KirigamiTable. KirigamiTable is a shape-changing tabletop that utilizes a kirigami actuator $[9,33]$ for shape changes. The prototype simulates a foldable touch display where output is generated via dynamic projection mapping, and display input is simulated via Wizard of $\mathrm{Oz}$ (i.e., remote touch events triggered via a tablet), allowing us to explore different touch-based interaction techniques. The design exploration is driven by a focus on supporting common proxemic transitions as seen in empirical studies of collaborative creative practices (e.g., [24]).

With the KirigamiTable prototype and a demonstration scenario ${ }^{1}$, we make the following contributions:

- A novel interface that supports collaboration around shapechanging tabletops

- Interaction techniques that combine transformations of shape and digital contents

- Demonstration of how kirigami actuation can be used to design a shape-changing tabletop

\section{RELATED WORK}

This work builds upon previous research on proxemics of collaborative work, display surfaces for co-located collaboration, and shape-changing interfaces.

\section{Proxemics, Collaboration and Transitions}

Proxemics is increasingly gaining traction in HCI due to the need for increased literacy regarding the interplay between collaborative activities, interactive technologies and spatial environments [17, 19, 38, 54, 50, 22, 71]. For instance, Interaction Proxemics [54, 50] and F-formations [34, 46, 71] are conceptual tools for articulating how interactive technology and the spatial environment together foster proxemic behaviours in collaborative work (i.e., hindering and enabling certain ways of acting in relation to others). F-formations describe spatial patterns in how people face each other through orientation and spacing between each other and artifacts [34, 46], e.g., a group of people may arrange in a semi-circular formation around a tabletop. In addition, a line of research focuses on transitions between proxemic relations [38, 71, 19]. Grønbæk et al.'s research on Proxemic Transitions highlights how stationary computers, laptops and furniture have several shortcomings in supporting transitions of entering and leaving collaborative situations, causing people to adapt to the environment rather than re-configuring it (e.g., groups huddle around personal displays or stand uncomfortably for sustained ad hoc meetings) [19]. Their work points to shape-changing furniture as a novel research direction for supporting people's re-configurations of the environment during such transitions.

\footnotetext{
${ }^{1}$ See the demonstration video accompanying this paper.
}

\section{Display Surfaces for Co-located Collaboration}

Research on collaborative surfaces has contributed prototypes and studies around different kind of surfaces, such as tabletop displays (e.g., [63]), wall displays (e.g., [56]), bended displays (e.g., [74]) and multi-display environments (e.g., [65, 11, 75]). It has been found that deciding which display form factor is best, depends on the activity it is used for [61, 59], e.g., vertical displays are well suited for coordination and presenting, whereas horizontal displays are better at supporting collaboration in terms of sharing ideas [59]. Empirical studies on collaboration have further shown that proxemic behaviour is supported by allowing for partitioning displays into territories, both on tabletops $[63,61,57,52,51]$ and wall displays $[30$, $47,73,56]$. For tabletops, in particular, it has been found that round- and square-shaped tables configure groups of people differently. For instance, round tables have shown to be problematic for some collaborative tasks due to their limited opportunities for partitioning the workspace as compared to rectangular tables [63]. Furthermore, the the ability to control orientation of objects and digital items on tabletops has been found to be crucial for how we collaborate $[39,25]$.

\section{Shape-Changing Interfaces}

Shape-Changing Interfaces is an emerging area of research. Up till now, it has predominantly focused on exploring smallscale applications (e.g. [76, 58, 72,31]) relating to the scale of handheld devices, some of which focus on combining digital displays and dynamic physical shape [3, 60, 45]. For instance, inFORM [13] demonstrates that rendering UI both digitally and physically allows for dynamic physical constraints.

Recent research on shape-changing interfaces has started to explore physically dynamic form at the scale of furniture (e.g., $[13,20,19,29])$. For instance, $2.5 \mathrm{D}$ displays have demonstrated how tabletops with a height dimension can enable new ways of interacting with multi-dimensional data $[44,43,10]$. These mostly focus on a new form of data display, and less on ways that the display shape may alter how groups can organize themselves spatially. Another strand of research focuses on addressing the technical challenges of constructing large-scale shape-changing objects (e.g., [55, 12, 62]), such as production methods for human-scale inflatable objects [62]. A few in-thewild studies have also demonstrated how changing the shape can mediate social interactions in new ways (e.g., $[20,53])$. Finally, and most relevant to our research, shape-changing furniture displays $[66,67,29,19]$ have been built to investigate their potential for supporting transitions in collaborative activities. These explore the relationship between transformations in shape, transitions in the activities they are used for, and collaborators' spatial organization enabled around them which Grønbæk et al. refer to as Proxemic Transitions [19].

While prior research on large-scale shape-changing displays $[67,19]$ points to future work of combining shape and digital content transformation, it remains largely unexplored.

\section{CHALLENGES FOR SHAPE-CHANGING TABLETOPS}

In the following, we highlight interaction design challenges for proxemic transitions based on prior findings in research on shape-changing interfaces and tabletop displays. 

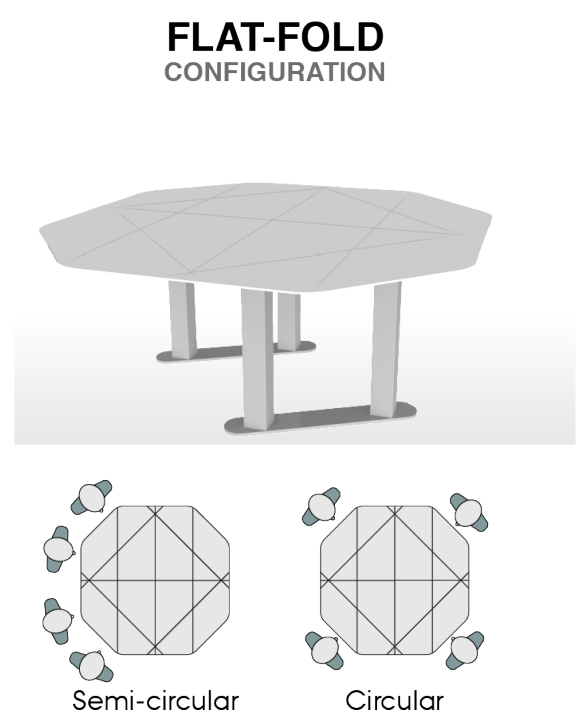

FOUR-FOLD

CONFIGURATION
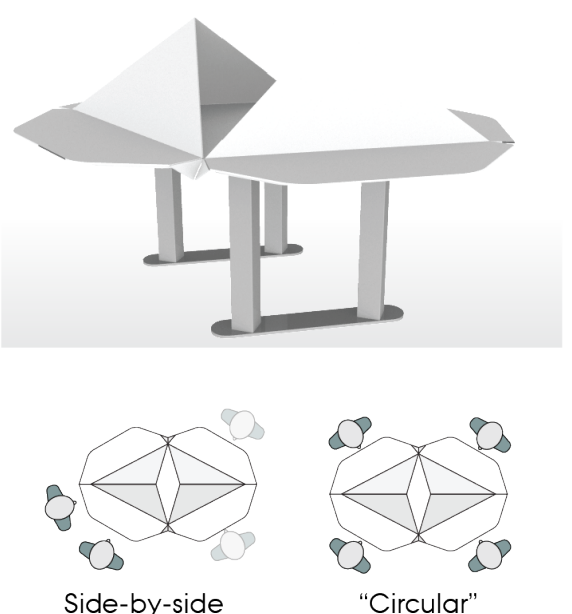

Side-by-side

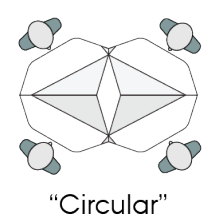

"Circular"
TWO-FOLD

CONFIGURATION
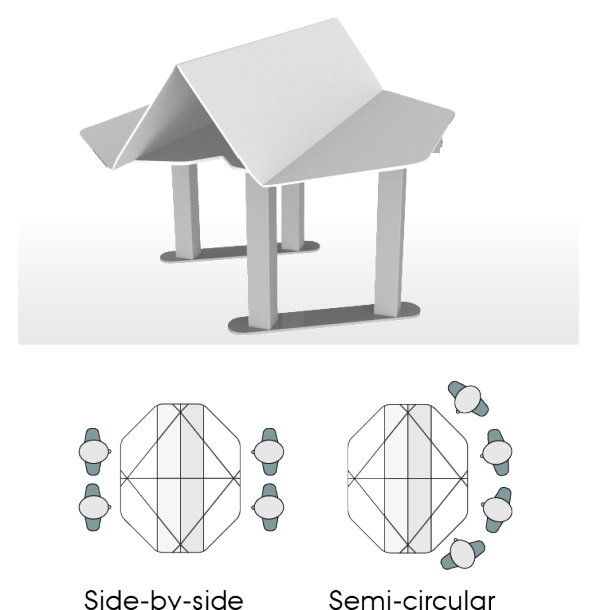

Semi-circular

Figure 2. KirigamiTable supports three types of shape configurations: Flat-Fold, Four-Fold and Two-Fold.

Transitions between Personal and Shared Work is a core challenge in tabletop research [16, 64, 27], and especially important for mixed-focus collaboration [21, 69, 67], e.g., brainstorming, affinity diagramming [4] and collaborative design [70]. Multi-device groupware often responds to this challenge by separating display territories between personal devices and tabletops $[68,77,48]$, e.g., facilitating transitions in collaborative activities by supporting branching, independent exploring and merging across devices [48].

Communicating Upcoming Shape-Changes is a challenge that has been addressed by enhancing shape changes with digital contents [60, 45, 13]. Lindlbauer et al. [45] propose different techniques that alter the optical appearance of dynamic objects for anticipating shape-changes.

Interaction with Large Shape-Changing Displays is in its early stages. Existing proposals can roughly be categorised as either implicit based on tracking of users' proximity relations (e.g., [66, 67]), or explicit interaction via physical buttons (e.g., [19]) or mid-air gestures (e.g., [67, 29]).

Supporting Cooperative Control is an important challenge for tabletops, because multiple users need to coordinate actions and resolve conflicts (e.g., [51, 69, 63, 27]). Studies further show that users are hesitant to collide in centralized controls (causing turn-taking) [51], and tend to not touch other people's personal territories [51, 63, 69].

\section{METHOD}

We explore interaction for Proxemic Transitions [19] with a kirigami-actuated shape-changing tabletop. Proxemic Transitions has served as a conceptual lens scoping the challenges identified in related work, identification of relevant activities for shape-changing tabletops, and finally, it has informed our design explorations by drawing attention to the dynamic interplay between social interaction, technology and furniture.

We have adopted a research-through-design methodology [14]. Through the construction of the actuated table and design of touch-based interactions simulated on the tabletop surface, we have been challenged to invent novel (preliminary) solutions, and as such, the prototype embodies a number of design choices that raise interesting design issues. In particular, we have explored how to move from the principle of kirigami actuation, as manifest in small-scale paper models (see Figure 15) to a proof-of-concept full-scale actuated tabletop surface (see Figure 2). Next, (in line with [7]) we explored design of interactions for proxemic transitions through a scenario (see Figures 3-5). We have focused on relevant scenarios from collaborative creative practices. This is a complex, challenging, and well-documented area of work, which is characterized by shifts in ways of collaborating and use of interactive technology to support work processes, and therefore puts our prototype design to a test. However, it should not be regarded as an exclusive application domain, and further potentially relevant areas are covered in the Discussion section. Finally, we synthesized the exploration into a set of interaction techniques for KirigamiTable (see Figures 6-14). Hence, the KirigamiTable prototype represents a point in a design space of shape-changing tabletops, inviting for further exploration.

\section{KIRIGAMI TABLE}

We envision a future where foldable shape-changing tabletop displays can enable transitions between different ways of working - together or individually. We explore this vision through KirigamiTable - a shape-changing tabletop augmented with digital projection (see Figure 1 and video). The table is capable of changing between three different shapes (see Figure 2), creating different socio-spatial conditions for working around it. The table surface utilizes a kirigami actuation principle [33, 9] to enable complex shape changes based on minimal actuation (using only two actuators operating along a single axis). See implementation section for more details on the prototype.

With the design of KirigamiTable, we explore how shapechanges can facilitate collaboration by enabling proxemic transitions between working individually, in small groups or large groups of people. 
The following three shapes of the table surface support different facing formations (see Figure 2):

- Flat-fold configuration can be used as any other tabletop, allowing the people in the group to move around the table surface freely.

- Four-fold configuration supports individual work, providing a semi-private work-area for each of the four members of the group.

- Two-fold configuration allows group members to work in pairs, while providing a shield space for each of the two pairs working at the table. It also provides a vertical display that may be desirable for presenting to others.
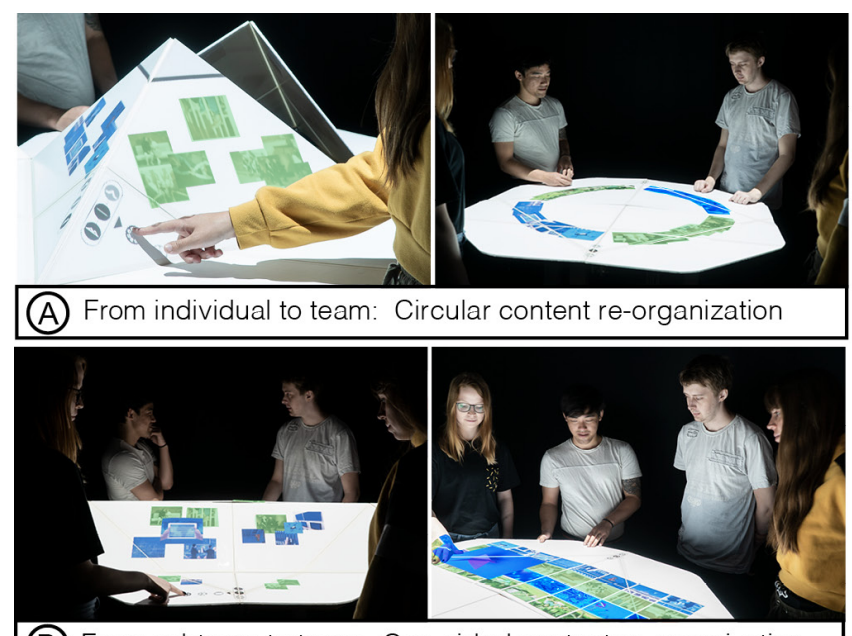

(B) From subteam to team: One-sided content re-organization
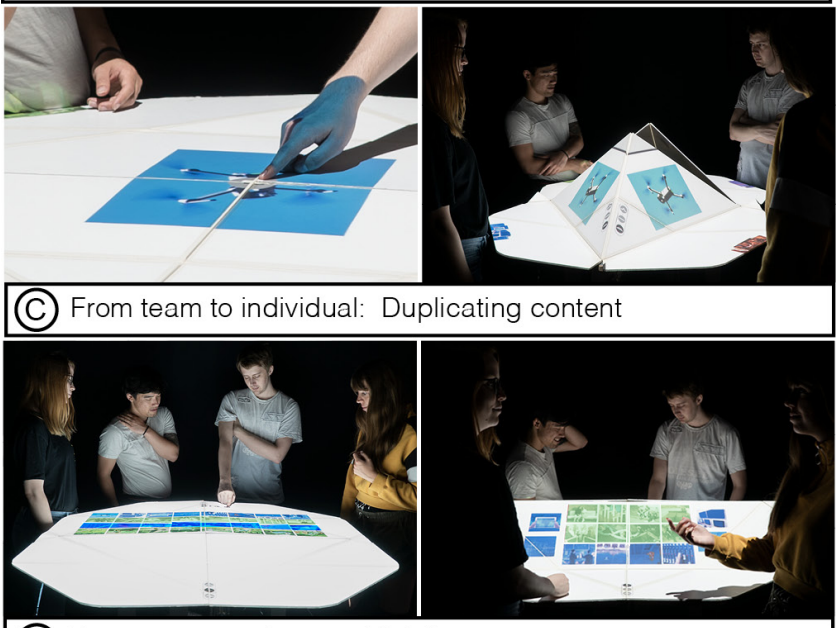

(D) From team to subteam: Dividing content

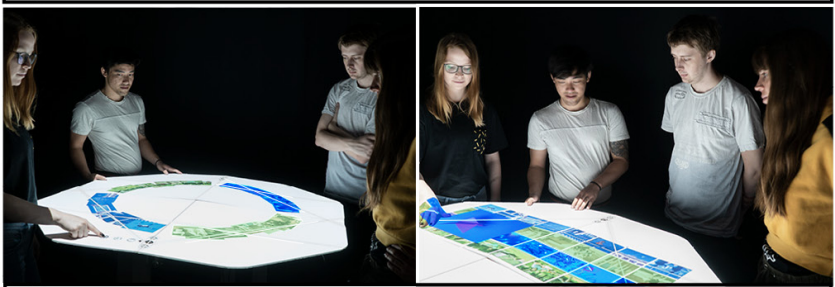

(E) Re-organizing content: From circular to one-sided

Figure 3. Basic Proxemic Transitions with KirigamiTable
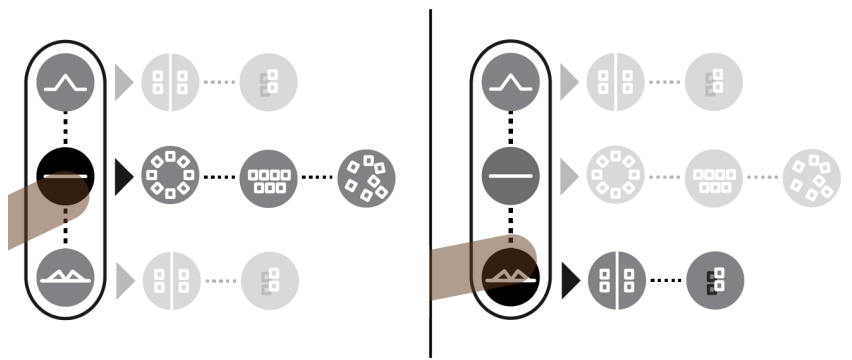

Figure 4. Control Widget: Users can slide along the vertical axis to expose menus of digital content re-organization for each table shape.

The range of proxemic (socio-spatial) relations illustrated for each shape in Figure 2 (indicated with F-formation patterns [34]) highlights an important point: we aim to empower people to dynamically re-organize around content in new ways. Hence, rather than making the tabletop dictate how people can re-organize through implicit interaction (e.g., via proxemic sensing $[66,67])$, shape-changes of KirigamiTable are initiated explicitly through touch-based interaction.

\section{Basic Proxemic Transitions via Control Widget}

Reflecting on shape-changes in the context of empirical observations of dynamic collaboration, Grønbæk et al. [19] highlight tradeffs for proxemic transitions: digital transformations enable small quick shifts in proxemic relations (such as scaling up a window on a big screen for a larger group to see), while physical transformations enable radical shifts which happen less frequently (such as collaborators re-organizing from a semi-circular to a circular formation). We build on this observation in our design proposals of combining physical shape and digital content transformations. In the following, we outline the basic proxemic transitions enabled by KirigamiTable (see Figure 3), showing how digital contents can be re-organized corresponding to the different shape transformations. We designed a control widget (see Figure 4) for basic interaction with shape-changes: users slide a finger along a vertical menu representing transitions between three possible shapes, where each shape-change supports different opportunities for re-organizing digital contents. The widget demonstrates the following basic content re-organizations:

- Transition to flat-fold: content items can be re-organized in circular, one-sided or maintain existing arrangement.

- Transition to two- and four-fold: a selection of content items can be divided or duplicated.

The widget is simplified to convey possibilities. Hence, it does not represent an exhaustive exploration of the different ways that content can potentially be re-organized along with shape-changes.

The proxemic transitions of Figure 3A-D all involve shapechanges, allowing for a shift in how people organize with spacing and orientation between each other. However, proxemic transitions can also simply be facilitated by re-organizing the digital content in the same shape configuration. For instance, the flat-fold configuration can support a range of facing formations, allowing people to re-organize the digital contents in a way that is appropriate for the current task (see Figure $3 \mathrm{E})$. The circular arrangement distributes contents around the 


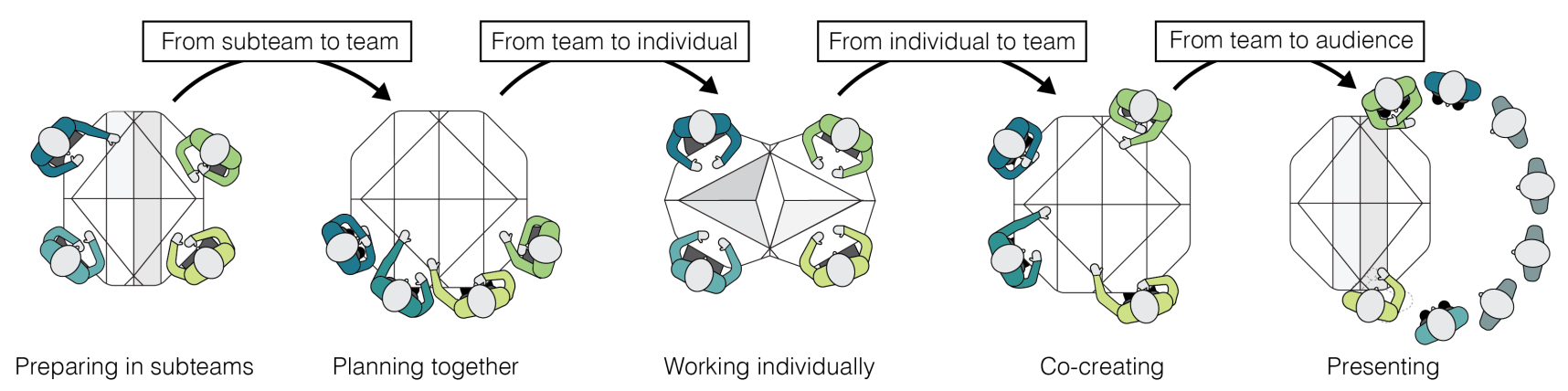

Figure 5. Scenario of proxemic transitions in creative collaborative practices: Inspiration Card Workshops (see also video figure)

surface oriented from a centre point, supporting a circular facing formation. However, there might be instances where it is desirable to re-organize from a circular to a semi-circular formation in order for everyone to view the digital contents from the same direction in a one-sided arrangement.

\section{Use Case: Collaborative Creative Practices}

Our design exploration with KirigamiTable is motivated and inspired by proxemic transitions related to collaborative creative practices. Several creativity methods take advantage of providing participants the opportunity to express themselves by both working individually (e.g., using Post-It notes), and sharing the materials with the rest of the team $[40,26,35,35$, $26,15])$. More generally, it is acknowledged that a hybrid structure, in which individuals first work independently and then work together generate better ideas than a team structure, in which the group works together throughout the whole process [15]. As a perspective on such transitions in team work, we draw from Grønbæk et al.'s distinction for Proxemic Transitions, where transitions manifest as either quick (and frequent) or radical (less frequent) shifts in people's social interactions. For instance, transitions in collaborative ideation may be subtle, such as shifting attention without particularly significant changes to interpersonal spacing and orientation (e.g., when co-creating via laptops [8]). However, as can be seen in the literature above, methods for collaborative creative practices often promote more disruptive shifts between individual and shared work, where materials need to be re-organized and developed over time. Such transitions may then manifest more significantly in people's spatial re-organization, as shown by Møller et al. in their study of collaborative ideation with physical and digital sticky notes [32]. While we have focused on proxemic transitions in the creativity domain, such transitions are not confined to these practices, but can also be found in, for instance, collaboration in a learning context [18], during surgery [50,49] or sense making [28, 5, 69].

In order to demonstrate the potential of KirigamiTable for supporting the more radical proxemic transitions, we have selected one of the many design methods which inherently incorporate dynamics between individual and collaborative activities: An Inspiration Card Workshop [24] is a collaborative design method involving professional designers and participants with knowledge of the design domain. As described in more detail in [24], the goal of such a workshop is to develop design concepts from two types of inspiration cards: Technology Cards and Domain Cards, index card-sized cards with a picture and a title. Figure 5 illustrates the common proxemic transitions and workflow as it unfolds around the KirigamiTable during the core part of an inspiration card workshop. Initially, the two sub-teams of domain experts and professional designers (technology experts) discuss domain cards and technology cards in sub-teams. Subsequently, they all convene in order to present the cards to each other. For the core part of the ideation process, participants for a short period typically work individually followed by co-creation in the team as a whole. The workshop is concluded by a presentation to other participants about the developed design concepts.

We implemented a basic application to explore how KirigamiTable may support common proxemic transitions in the collaborative practice of inspiration card workshops (see Figure 3): content items in the Kirigami UI are inspiration cards of images and videos, which are color-coded in two separate categories - blue for technology cards and green for domain cards - to illustrate the basic distinctions between users working in subteams (separated colors) and when they all collaborate and combine cards (mixed colors).

\section{INTERACTION TECHNIQUES FOR KIRIGAMITABLE}

Having described the design of the KirigamiTable, the basic proxemic transitions it enables and an application scenario, we now describe the results of our explorations into designing interaction techniques for the KirigamiTable. These explorations have been guided by the challenges of designing for proxemic transitions with a shape-changing tabletop that we earlier identified in terms of how to design for initiation and anticipation of shape-changes as well as cooperative control.

\section{Initiating Shape and Content Transformations}

Apart from the basic interactions with KirigamiTable, our design work opened up a range of opportunities for how shape transformation can possibly trigger meaningful content transformations on a shape-changing surface, and vice-versa.

\section{Shape-First Interaction}

The basic transitions (see Figures 3 and 4) demonstrate interaction through a control widget, where physical shape transformation is taken as the primary entry point for interaction, and users subsequently decide how the digital contents should be re-organized and distributed across the surfaces. We refer to this technique as shape-first interaction, because it starts with 


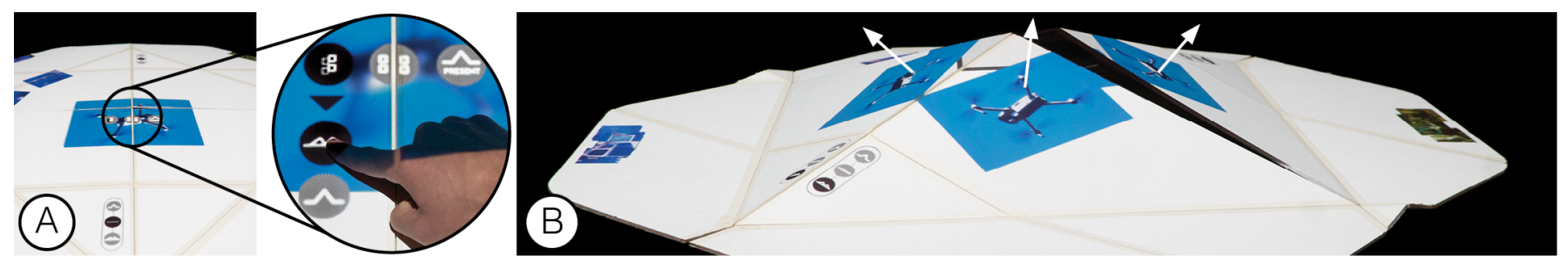

Figure 6. Content-first widget: A card is selected for duplication, and subsequently the four-fold shape is selected (A). KirigamiTable splits into four-fold with four duplicates of the card, provided on each individual vertical surface in the four-fold configuration (B).
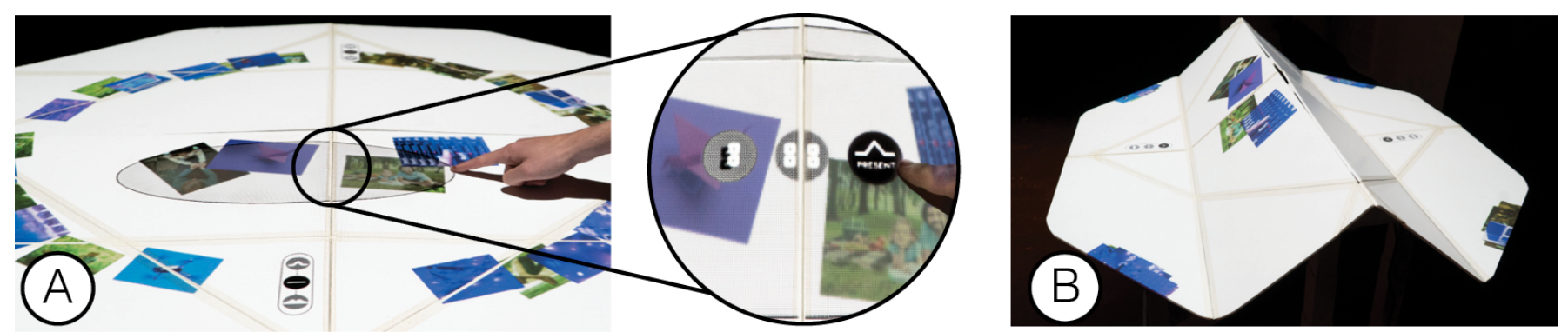

Figure 7. Content-first widget: A widget emerges from a selection action, indicating possibilities for content manipulations that also trigger shapechanges (A). Presentation mode is selected, and the table transforms into two-fold (B)

a menu of shape-changes, followed by a sub-menu of options for re-organizing contents according to the shape-change.

\section{Content-First Interaction}

An alternative approach is to trigger shape-changes implicitly, based on digital content manipulations. This is referred to as content-first interaction. When a user selects one or more cards, a content-first widget appears that operates on the selection. For instance, the user can choose to duplicate a card into four individual copies, triggering a shape-change that corresponds to the content action, as illustrated in Figure 6. Additionally, a user can choose a selection of cards for presentation as illustrated in Figure 7, subsequently triggering a shape-change into the two-fold with vertical display real-estate for presenting to others.

\section{Cooperative Control of Shape-Change}

The design of cooperative control with KirigamiTable builds on prior tabletop research $[51,27]$ that responds to the challenge of designing multi-user interactions for tabletops. First, Morris et al. [51] propose coordination policies for handling conflicts of control, e.g., touching the same item may duplicate the item or tear it to pieces, or cooperative gestures may serve as a mechanism to avoid potentially disruptive actions to occur by accident. Second, Hornecker [27] exploits embodied constraints of tabletops (caused by their size and shape) to explicitly inhibit certain social behaviours (e.g., control conflicts) while inducing others by design (e.g., helping and participation). Based on these findings, we propose two techniques for designing controls for shape-changing tabletops: dynamic distribution of controls and cooperative gestures.

\section{Dynamic Distribution of Controls}

As part of designing the shape-first interaction, we considered coordination problems of interaction. It has been proposed to exploit (or even overcome) embodied constraints on action around tabletops [27, 51, 63]. For instance, Morris et al. show that providing private feedback locally in the user's territory can avoid social embarrassment [51], and Hornecker shows that providing multiple access points for control increases participation [27].

With KirigamiTable, we demonstrate that physical tabletop transformations can change how digital territories are physically constrained, in consequence making some actions possible and inhibiting others (e.g., flat-fold facilitates social awareness and equal participation, while four-fold facilitates individual focus). However, since the four-fold configuration supports individual display territories, it also complicates shared control of shape-changes because no-one can see the entire display surface. Hence, we propose to provide multiple access points [27] for interacting with shape-change (see Figure 8): control widgets are dynamically re-distributed depending on the shape to continually provide multiple access points that are accessible and visible from any position around the display.

\section{Cooperative Gestures for Shape-Change}

An important challenge for collaborative displays is to design interactions that avoid potentially disruptive actions to occur by accident [51]. The potential of causing disruptive effects to people's work environment is specifically evident with shapechanging tabletops, in the sense that when shape-changes occur it radically transforms the space around which people work. This is further exacerbated when interactions with shape-

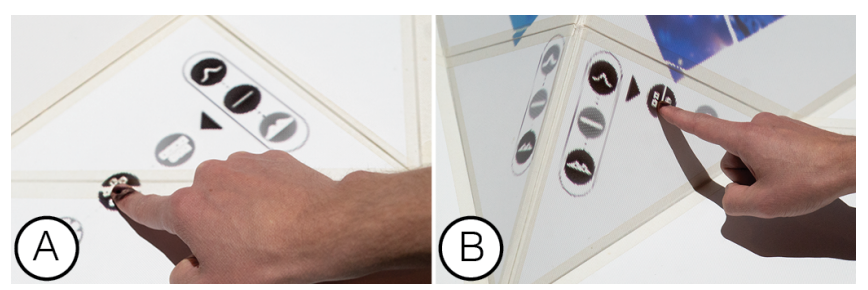

Figure 8. Control widgets are distributed depending on the shape: in two-fold and flat-fold there is one widget on each end $(\mathrm{A})$, while in fourfold each personal area has a widget (B). 
changes are implicit, i.e., based on contextual information (such as in content-first interaction).

A response to this challenge, is seen in Morris et al.'s concept of cooporative gestures [51]. Building on this concept, we explore how cooperative gestures can be designed for KirigamiTable: Figure 9 demonstrates a cooperative gesture, where the four participants collaboratively trigger a duplicate action of an item by all simultaneously touching the same inspiration card. The number of hands detected for the gesture can then determine whether to transform into four-fold (four hands) or two-fold (two hands).

\section{Anticipating Shape-Changes}

One obvious challenge is how to provide feedforward regarding the physical shape transformation of an actuated surface. As shape-changes are disruptive to the ongoing activity, dynamic displays can provide feedforward on their upcoming shape-change. In the following, we illustrate how prior ideas of feedforward at handheld shape-changing device scale [58, 45] can be extended to furniture scale and support collaborators in anticipating shape-changes for coordinating their actions around the tabletop.

\section{Digital Preview: Indicating via Digital Animation}

Lindlbauer et al. [45] propose the concept of Anticipating shape-change, where upcoming shape-changes are simulated virtually by altering the optical appearance of shape-changing objects with displayed graphical animation. We extend this concept for collaborative tabletops with KirigamiTable (see Figures 10 and 11). Here, digital previews are not only hinting at changes, but can also indicate how content will be re-organized. For instance, when transforming from team to individual work, the interface may first indicate how content is sub-divided before it shape-changes (see Figure 10).

\section{Physical Preview: Indicating via Intermediate Shape Steps}

Rasmussen et al. [58] propose the concept of Negotiated control of shape-change (for single-user interactions), where shape-change can be negotiated (i.e., initiated and altered) between the user and the interface. We explore this principle for designing (multi-user) interactions at tabletop scale: physical previews use intermediate shape steps (i.e., a slight shapechange hinting at a full shape-change) to provide feedforward on a global interface transformation affecting all users' individual activities. As shown in Figure 12A-B, a slight shape change towards an intermediate step can communicate an upcoming shape change and prompt users for preparing their cards (sorting into private and shared) for the transition. In

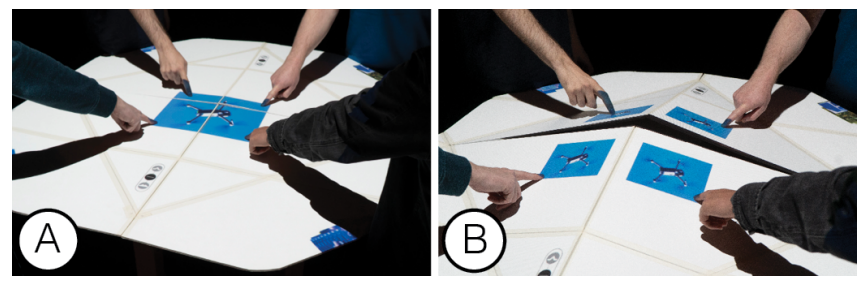

Figure 9. Cooperative gesture: Four hands simultaneously pulling away from the centre of the surface (A) will implicitly trigger a duplicate action, transforming into four-fold $(B)$.

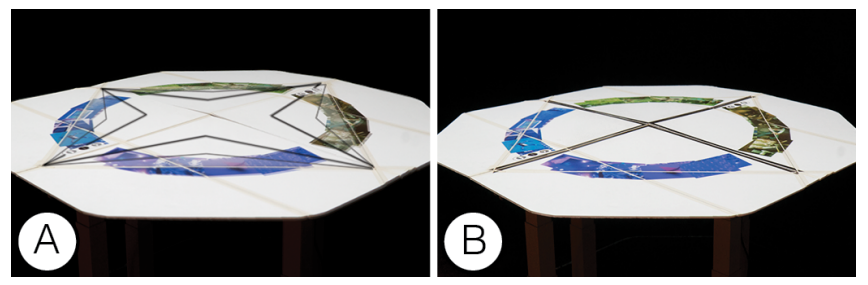

Figure 10. Digital preview (four-fold): indicating transitions through digital animations that resemble the shape change (A). The folds are highlighted digitally before the shape change (B).

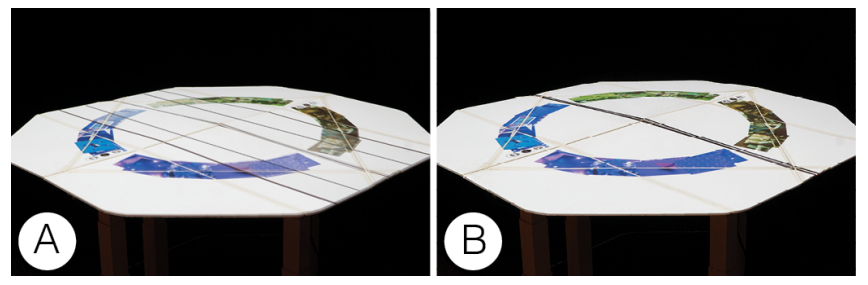

Figure 11. Digital preview (two-fold): indicating transitions through digital animations that resemble the shape change (A). The fold is highlighted digitally before the shape change (B).

the current demonstration, the intermediate period is timerbased. However, it could as well prompt users to cooperatively confirm the shape-change when they have sorted their cards.

\section{Highlighting Edges that Fold}

A previewing technique that is utilized in both of the others is to digitally highlight the folding edges. This is not only to indicate how the shape is going to fold in an upcoming transition, but can also serve as a digital boundary for indicating a threshold between private and shared or how contents will be split between individuals.

First, we implemented a digital boundary as a draggable bar (see Figure 13), enabling users to prepare before triggering transformations from individual to team work: users can sort cards into private and shared via the boundary separating the contents on the triangle UI. This is input to the table transformation when re-organizing content for a different shape configuration. However, in developing this transition, we further explored the idea that highlighting a subset of edges on the kirigami pattern can indicate boundaries during transitions. We extended the draggable bar to implicitly trigger a shapechange to flat-fold when dragging over the physical boundary of a shape fold (see Figure 14A-B). After the trigger, the folding edges below each triangle in the four-fold configuration are highlighted digitally to indicate to everyone that a transition is coming up, and they can prepare by sorting cards into share and minimize zones (see Figure 14C).

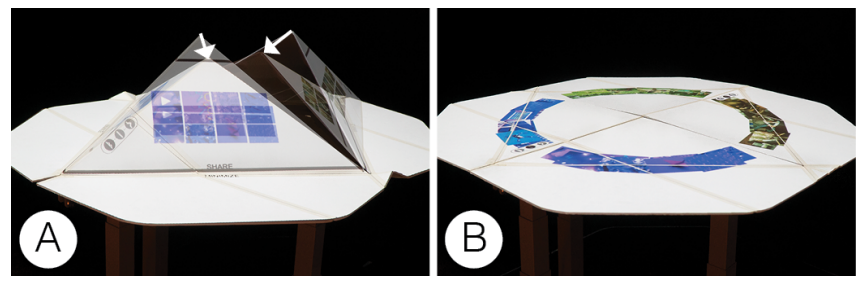

Figure 12. Physical preview: indicating a transition towards flat-fold by an intermediate step (A), prompting users to finalize their individual card sorting activities. It subsequently transforms fully into flat-fold (B). 


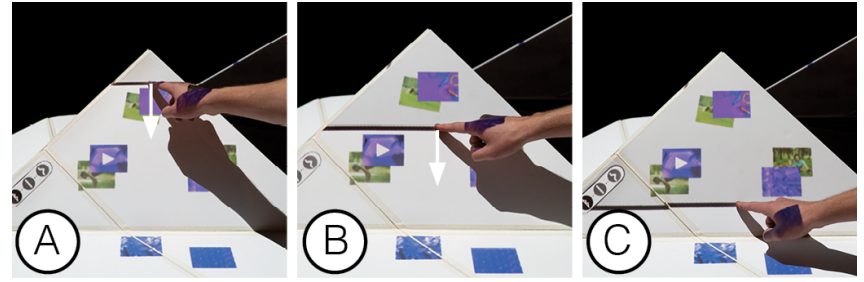

Figure 13. Before shape change, a digital boundary allows for preparing content, dividing it into content that will be shared and minimized (A). The boundary is draggable (B-C).

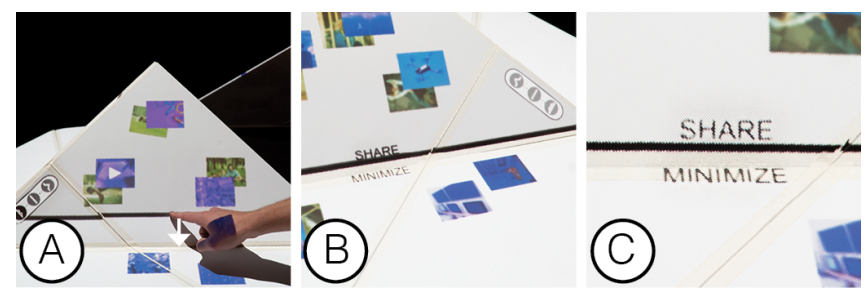

Figure 14. Shape change is triggered implicitly based on dragging the digital boundary below the physical fold on the shape (A). Fold edges all around the table are highlighted (B) to indicate a boundary between what is will be shared and minimized, respectively (C).

\section{DESIGN AND IMPLEMENTATION}

The following outlines the design of the Kirigami surface and describes the technical implementation of the different components of the KirigamiTable prototype.

\section{The Kirigami Surface}

The surface of the table utilizes a metamaterial strategy called Kirigami Actuators [9, 33], in order to create complex threedimensional transformations through a simple linear actuation. Kirigami Actuators build on the traditional Japanese art of Kirigami, where folding and cutting paper is used to create three-dimensional structures. Dias et al. [9] have illustrated how understanding the mechanical properties of thin sheets makes it possible to generate four fundamentally different forms of actuation: roll, pitch, yaw, and lift, using the same linear actuation. Building on this work, Kaspersen et al. [33] further explored how the same principles can be utilized when thickness is added to thin sheets. The Kirigami surface utilized in the design builds on the foundational work of Dias et al. [9], and utilizes some of the tactics proposed by Kaspersen et el.

The starting point for the design process of the Kirigami surface was to explore different ways that the surface actuation can be controlled. Figure 17 illustrates how creating a simple cut down the centre of a piece of paper, allows for an up- or downwards actuation of the surface when it is pulled, creating a soft curve on the surface. By introducing folds into the surface (see Figure 15), the direction and placement of the actuation can be controlled. Figure 15 illustrates how different aspects of this basic principle have been explored during the design process. This includes different patterns of cuts and folds, and combinations of patterns, different shapes of the table (eclipse, rounded square), and different combinations of surface materials (cardboard \& tape, wood \& textile, 3-d printing on textile, gluing laser cut elements on textile). In the process different shapes and proportions of the surface have been experimented with, to achieve a surface that works in each of the three shape configurations and where users have equal access from all sides.

We sought specific inspiration from common proxemic transitions that occur in collaborative creativity methods such as Inspiration Card Workshops when generating design ideas. Finally, using the actual shape (affordances and constraints) of the Kirigami Actuator as inspiration for generating UI concepts, we approached the challenges of designing a segmented UI that consisted of, not just rectangles, but also triangles and bends with unconventional UI edges. This further led to the idea of using its transitions from flat-fold to the other shapes as a metaphor for 'splitting' content.

Based on these design considerations, the KirigamiTable surface is constructed out of individual cardboard segments, which are taped together to create hinges. Figure 17 illustrates the different components of the surface; hinges with valley and mountain folds as well as cuts.

\section{The Actuation and Frame}

Figure 16 illustrates the construction of the frame and actuation mechanism of KirigamiTable. The frame underneath the table consists of four linear actuators (Linak DL17) for height-shifting the table between a seated to a standing table. To change the shape of the table, two linear actuators (Linak LA14) actuate from the centre and outwards. Two rails have
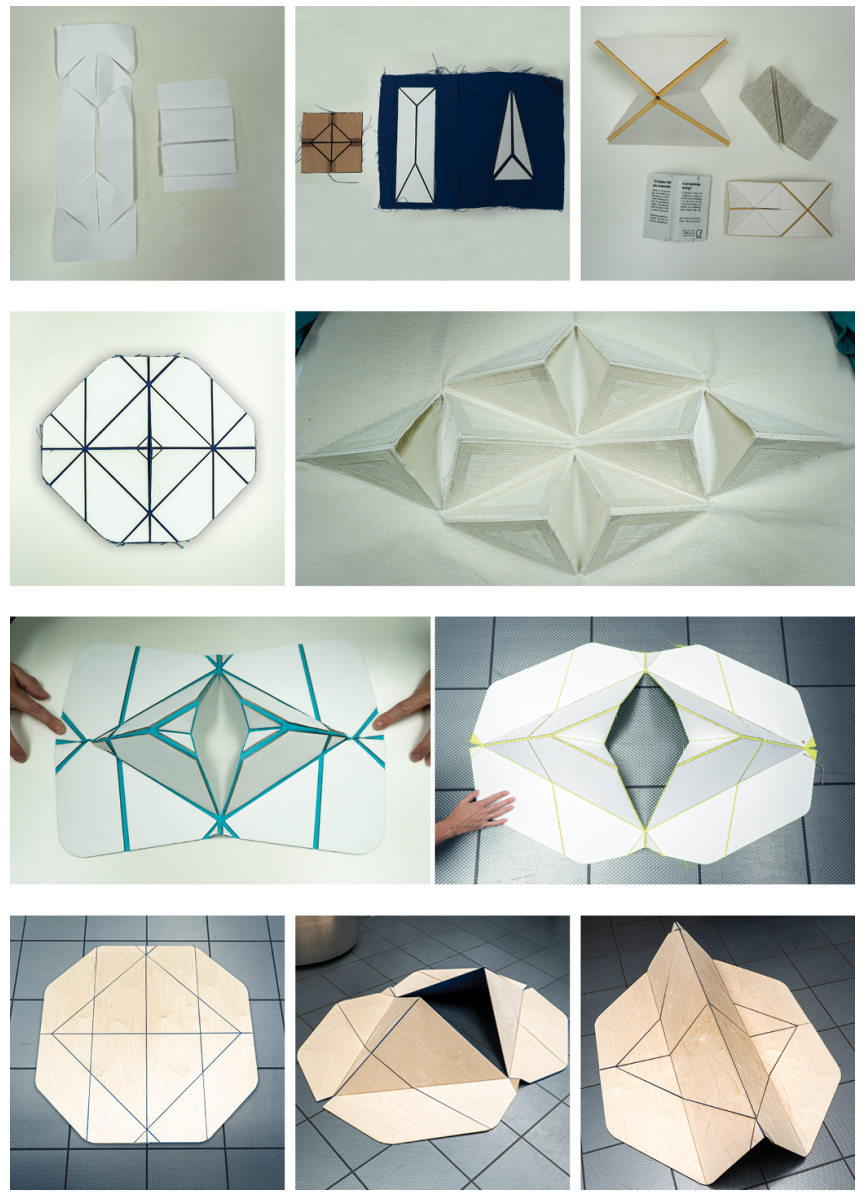

Figure 15. Prototypes of different kirigami patterns. 
been used to create an attachment point on a central axis and to support the actuation. In principle, the surface actuation could have been carried out by a single actuator, but to maintain the symmetry along a central axis, allowing the table to expand from a central point, two actuators have been used. The Kirigami surface rests on a wooden surface mounted on top of the frame, and is connected at two points to the actuator.

\section{The Projection Mapping Setup}

Projection mapping is achieved using a single projector mounted over the table. A virtual 3D model of the Kirigami surface is rigged inside Unity and the Unity physics system is used to simulate the physical deformations virtually, and custom-designed calibration software developed by our research laboratory $C A V I$ [23] enables the digital model to be projected precisely on the Kirigami surface according to the physical transformation of the table. Unity receives events for virtual deformation through a USB interface to the Linak actuators. The actuated state is continuously communicated as events with a new integer value from the actuators, indicating its current actuation along one linear axis. These events can then be applied as a virtual linear force along the same axis in the virtual representation, consequently resulting in a virtual deformation that resembles the physical deformation.

\section{The Interactive Application Setup}

A host PC runs the Unity application and displays it through the projector. The Unity application is responsible for rendering the graphics using the estimated projector pose from the calibration. The surface of the 3D model renders a texture with the real-time updated contents of a Chromium browser, allowing us to embed a Web application on the Kirigami surface. The same host PC runs an event bus application, responsible for USB communication with the Linak USB interface and WebSocket communication to the Web app running inside Unity. The Web application is implemented using Webstrates [37], which synchronizes DOM events between open browser clients. Webstrates thus serves as infrastructure for Wizard Of $\mathrm{Oz}$, enabling a tablet to remotely trigger multi-touch events in the Web app, which in the Unity Web container translate into both 2D content manipulations on the projected Kirigami surface and table events that actuate the table surface.

\section{DISCUSSION}

Through the design and implementation of the KirigamiTable, we have come to identify a set of challenges and opportunities for shape-changing tabletops that point towards future work for this new and exciting type of user interfaces.

\section{Designing Interactions for Proxemic Transitions}

Designing interactions for proxemic transitions with a shapechanging tabletop is complex. With the KirigamiTable, we have demonstrated a set of novel interaction techniques, exploring how shape-change and re-organization of digital contents can provide new opportunities for meaningful proxemic relationships in collaboration around a tabletop interface. However, these techniques should not be considered as final solutions, but rather as initial explorations. With the control widgets, we have, in particular, presented shape-first and contentfirst interaction techniques as different approaches to initiating shape-changes. But there are rich possibilities for combining shape and content transformation and this topic invites for further investigation. There may be a potential in exploring different forms of negotiated shape-change [58], e.g., where content and shape are manipulated in parallel, either in a stepwise re-configuration of shape and digital content [19], or perhaps by concurrent, continuous manipulations. In addition, the demonstrated control widget only conveys a preliminary mapping of shape-change and content transformation.
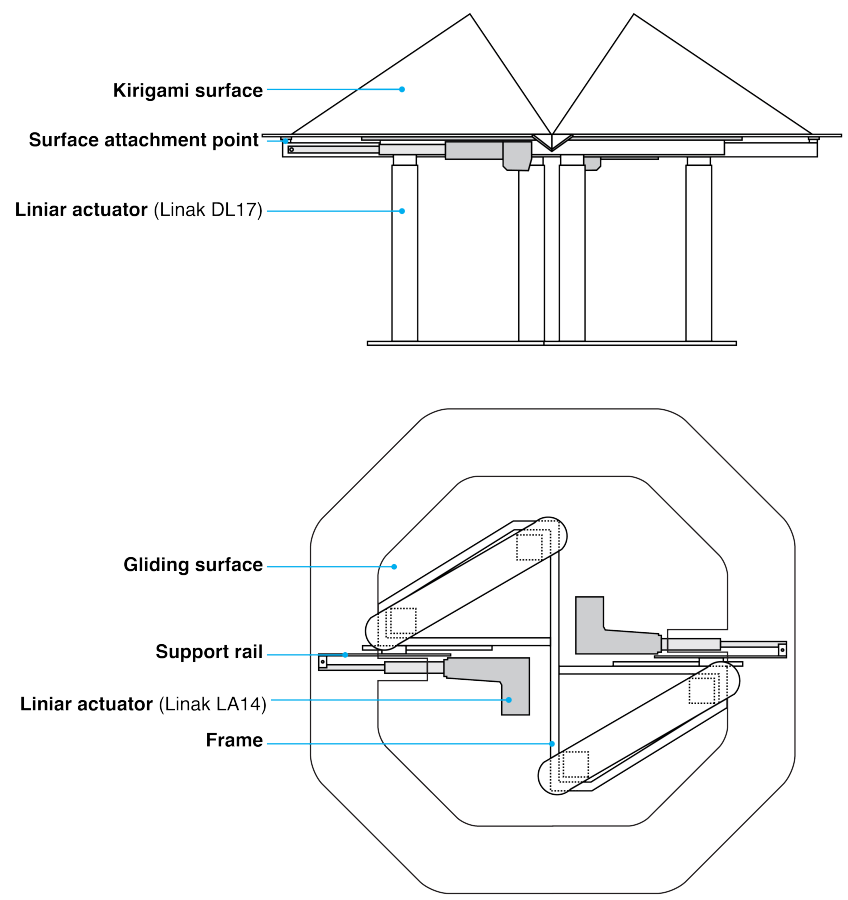

Figure 16. Technical drawing of the Kirigami table, top: side view of table, below: view from bottom up.

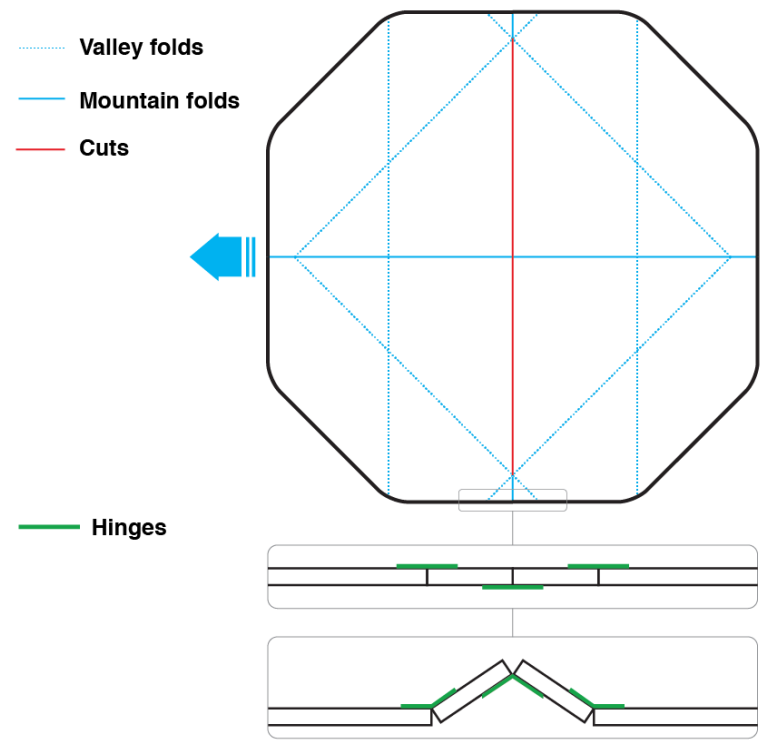

Figure 17. The Kirigami surface. Top: top view of kirigami surface. Bottom: section side view of hinge structure 
Our particular design choices then serve as inspiration for the research community, inviting for more systematic exploration and mapping of possible combinations of shape and content transformation - including future evaluations of these.

\section{Automatic Re-organization of Contents}

One of the obvious design challenges with a shape-changing tabletop is that, when shape-changes occur, contents often need to be re-organized to accommodate for the new shape configuration. This is a challenge since the ability to manage orientation of shared objects is crucial for how we collaborate $[39,25]$, and it has been shown that no single digital orientation and translation mechanism is best for every scenario of interaction in collaboration, however, each presents its tradeoffs [25]. It is further complicated by the fact that organization of digital items on KirigamiTable is not merely a matter of pure $2 \mathrm{D}$ translation and orientation. The digital display is only consistently flat in the flat-fold configuration, however, in both of the other configurations, there are multiple horizontal and vertical parts on the display surface. This creates a complex interplay between shape and displayed contents, which only adds to the potential confusion in perceiving contents that are being re-organized along with shape-changes. However, as there are many possible ways that contents can be meaningfully re-organized (as well as confusingly re-organized), we suggest that the menu options are scoped by designers for particular applications with widgets and controls that only provide action possibilities meaningful to a particular activity.

\section{Transformations with Global Consequences}

We have proposed the interaction techniques of digital and physical previews to align expectations among users regarding transformation with "global" consequences (i.e., consequences for everyone using it). There are trade-offs when comparing digital and physical previews. Physical previews make good sense when not all users can see the entire display (as in fourfold) because the change of a shape is more visible to everyone. However, digital previews work well for transitions from flatfold, because the entire display surface is visible to everyone - and in case objects like cups are resting on the table, they can be safely handled before the transition. Furthermore, a consequence of the kirigami pattern of the KirigamiTable is that, when the shape changes, it changes for everyone around the table. This is in contrast to TRANSFORM $[42,29]$ which enables "local" shape-changes. KirigamiTable, then, has its limitations with respect to the proxemic transitions that it supports. However, it would be feasible to design a different structure, e.g. with multiple, local kirigami structures.

\section{Potential Application Areas}

As aforementioned, the kirigami pattern on KirigamiTable mostly facilitates radical shifts [19], in that the transformations have consequences for everyone around the tabletop. We believe that this is most appropriate in situations, where synchronous shifts among everyone around the table is relevant. This could be in structured processes such as collaborative creative practices, but it could also be in educational settings, where it would be relevant for pedagogic reasons to facilitate certain proxemic transitions. Informal feedback from teachers and educators based on demonstrations of the KirigamiTable have pointed to its potential for facilitating group activities in classrooms. Furthermore, considering different time frames points to other potential application areas. In the Inspiration Cards scenario, we have focused on transitions happening within minutes or hours, however, it could also be relevant to consider transitions over several hours or days. This could be classrooms or other public and private spaces that are used in different ways throughout the day.

Finally, while prior research on Collaborative Coupling Styles (around tabletops [69, 28] or across devices [5]) exhibit less radical proxemic transitions, it can inspire future work for exploring how the combination of shape-changing tabletops and people's mobile devices can support Proxemic Transitions.

\section{Limitations of the KirigamiTable Prototype}

An important next step is to evaluate shape-changing tabletops with users in collaborative activities. The KirigamiTable prototype has certain limitations which prevent us from further evaluating its potentials. In particular, the touch functionality on the tabletop is simulated in the current prototype. In order to conduct user studies, we need to further develop KirigamiTable into a fully interactive prototype. This involves developing and integrating a multitouch-enabled display surface. As the first foldable touch-enabled OLED displays have already been produced (e.g., [41,36]), we are not far from being able to cover shape-changing tabletops with OLED displays. And we believe that kirigami principles provide an interesting approach to enabling this future, in that an actuated foldable display surface ideally can be manufactured from one or two ultra-thin foldable display films that can cover the two sides of the cut in the kirigami pattern.

\section{CONCLUSION}

We presented the design of KirigamiTable - a shape-changing tabletops for supporting transitions in co-located collaboration. Our work has started to unpack the potentials of emerging interactions that combine physical shape change and digital content re-organization to enable new ways of transitioning between working individually and collaboratively. Our research-through-design process has highlighted a set of novel touch-based interaction techniques for supporting proxemic transitions: (1) Initiating Content and Shape Transformations (shape-first and content-first interaction), (2) Supporting Cooperative Control (dynamically distributed controls and cooperative gestures), and (3) Anticipating shape-changes (digital previews, physical previews and highlighting edges). Our summary of novel opportunities and challenges with shapechanging tabletops outline an exciting new direction for supporting transitions in co-located collaboration via interaction with the combination of shape-change and digital contents.

\section{ACKNOWLEDGMENTS}

This work was supported by Microsoft Research through its PhD Scholarship Programme and the CIBIS project [1]. The prototype is built with custom-made software by CAVI [23] and custom-made hardware by LINAK [2]. Thanks to CAVI intern Sebastian Krog Knudsen for making the stunning video. 


\section{REFERENCES}

[1] 2019. CIBIS - Creativity in Blended Interaction Spaces. (20 12 2019). Retrieved from

https://cavi.au.dk/projects/cibis/.

[2] 2019. LINAK - electronic linear actuator technology systems for movement. (20 12 2019). Retrieved from https://www.linak.com/.

[3] Jason Alexander, Andrés Lucero, and Sriram Subramanian. 2012. Tilt Displays: Designing Display Surfaces with Multi-Axis Tilting and Actuation. In Proceedings of the 14th International Conference on Human-Computer Interaction with Mobile Devices and Services (MobileHCI '12). Association for Computing Machinery, New York, NY, USA, 161-170. DOI : http://dx.doi.org/10.1145/2371574.2371600

[4] Hugh Beyer and Karen Holtzblatt. 1997. Contextual Design: Defining Customer-Centered Systems. Elsevier. Google-Books-ID: JxQaQgOONGIC.

[5] Frederik Brudy, Joshua Kevin Budiman, Steven Houben, and Nicolai Marquardt. 2018. Investigating the Role of an Overview Device in Multi-Device Collaboration. In Proceedings of the 2018 CHI Conference on Human Factors in Computing Systems (CHI'18). ACM, New York, NY, USA, Article 300, 13 pages. DOI : http://dx.doi.org/10.1145/3173574. 3173874

[6] W. Buxton. 2006. Space-function integration and ubiquitous media. Towards an Interactive and Integrative Design Process. Linz, Austria: DOM Publications (2006), 248-271.

[7] John M Carrol. 1999. Five reasons for scenario-based design. In Proceedings of the 32nd Annual Hawaii International Conference on Systems Sciences. 1999. HICSS-32. Abstracts and CD-ROM of Full Papers. IEEE, 11-pp.

[8] Bo T Christensen and Sille Julie J. Abildgaard. 2018. Co-Located Team Designing: the oscillation between individual and social processes. In Design Research Society 2018. Design Research Society, 1297-1313.

[9] Marcelo A Dias, Michael P McCarron, Daniel Rayneau-Kirkhope, Paul Z Hanakata, David K Campbell, Harold S Park, and Douglas P Holmes. 2017. Kirigami actuators. Soft matter 13, 48 (2017), 9087-9092.

[10] Aluna Everitt and Jason Alexander. 2017. PolySurface: A Design Approach for Rapid Prototyping of Shape-Changing Displays Using Semi-Solid Surfaces. In Proceedings of the 2017 Conference on Designing Interactive Systems (DIS '17). Association for Computing Machinery, New York, NY, USA, 1283-1294. DOI :

http://dx.doi.org/10.1145/3064663.3064677

[11] Katherine Everitt, Chia Shen, Kathy Ryall, and Clifton Forlines. 2006. MultiSpace: Enabling electronic document micro-mobility in table-centric, multi-device environments. In First IEEE International Workshop on Horizontal Interactive Human-Computer Systems (TABLETOP'06). IEEE, 8-pp.

[12] Sean Follmer, Daniel Leithinger, Alex Olwal, Nadia Cheng, and Hiroshi Ishii. 2012. Jamming User Interfaces: Programmable Particle Stiffness and Sensing for Malleable and Shape-Changing Devices. In Proceedings of the 25th Annual ACM Symposium on User Interface Software and Technology (UIST'12). Association for Computing Machinery, New York, NY, USA, 519-528. DOI :

http://dx.doi.org/10.1145/2380116.2380181

[13] Sean Follmer, Daniel Leithinger, Alex Olwal, Akimitsu Hogge, and Hiroshi Ishii. 2013. InFORM: Dynamic Physical Affordances and Constraints through Shape and Object Actuation. In Proceedings of the 26th Annual ACM Symposium on User Interface Software and Technology (UIST'13). Association for Computing Machinery, New York, NY, USA, 417-426. DOI : http://dx.doi.org/10.1145/2501988.2502032

[14] William Gaver. 2012. What Should We Expect from Research Through Design?. In Proceedings of the SIGCHI Conference on Human Factors in Computing Systems (CHI'12). ACM, New York, NY, USA, 937-946. DOI :

http://dx.doi.org/10.1145/2207676.2208538

[15] Karan Girotra, Christian Terwiesch, and Karl T Ulrich. 2010. Idea generation and the quality of the best idea. Management science 56, 4 (2010), 591-605.

[16] Saul Greenberg, Michael Boyle, and Jason LaBerge. 1999. PDAs and shared public displays: Making personal information public, and public information personal. Personal Technologies 3, 1-2 (1999), 54-64.

[17] Saul Greenberg, Nicolai Marquardt, Till Ballendat, Rob Diaz-Marino, and Miaosen Wang. 2011. Proxemic Interactions: The New Ubicomp? Interactions 18, 1 (Jan. 2011), 42-50. DOI :

http://dx.doi.org/10.1145/1897239.1897250

[18] Jens Emil Grønbæk, Mille Skovhus Knudsen, Kenton O'Hara, Peter Gall Krogh, Jo Vermeulen, and Marianne Graves Petersen. 2020. Proxemics Beyond Proximity: Designing for Flexible Social Interaction Through Cross-Device Interaction. In Proceedings of the 2020 CHI Conference on Human Factors in Computing Systems (CHI '20). ACM, New York, NY, USA. DOI : http://dx.doi.org/10.1145/3313831.3376379

[19] Jens Emil Grønbæk, Henrik Korsgaard, Marianne Graves Petersen, Morten Henriksen Birk, and Peter Gall Krogh. 2017. Proxemic Transitions: Designing Shape-Changing Furniture for Informal Meetings. In Proceedings of the 2017 CHI Conference on Human Factors in Computing Systems (CHI '17). Association for Computing Machinery, New York, NY, USA, 7029-7041. DOI :

http://dx.doi.org/10.1145/3025453.3025487 
[20] Erik Grönvall, Sofie Kinch, Marianne Graves Petersen, and Majken K. Rasmussen. 2014. Causing Commotion with a Shape-Changing Bench: Experiencing Shape-Changing Interfaces in Use. In Proceedings of the SIGCHI Conference on Human Factors in Computing Systems (CHI '14). Association for Computing Machinery, New York, NY, USA, 2559-2568. D0I : http://dx.doi.org/10.1145/2556288.2557360

[21] Carl Gutwin and Saul Greenberg. 2002. A descriptive framework of workspace awareness for real-time groupware. Computer Supported Cooperative Work (CSCW) 11, 3-4 (2002), 411-446.

[22] Edward Twitchell Hall. 1966. The hidden dimension. Garden City, NY: Doubleday.

[23] Kim Halskov. 2011. CAVI: An Interaction Design Research Lab. Interactions 18, 4 (July 2011), 92-95. DOI : http://dx.doi.org/10.1145/1978822.1978841

[24] Kim Halskov and Peter Dalsgård. 2006. Inspiration Card Workshops. In Proceedings of the 6th Conference on Designing Interactive Systems (DIS '06). ACM, New York, NY, USA, 2-11. DOI :

http://dx.doi.org/10.1145/1142405.1142409

[25] Mark S Hancock, Frédéric D Vernier, Daniel Wigdor, Sheelagh Carpendale, and Chia Shen. 2006. Rotation and translation mechanisms for tabletop interaction. In First IEEE International Workshop on Horizontal Interactive Human-Computer Systems (TABLETOP'06). IEEE, 8-pp.

[26] Bruce Hanington and Bella Martin. 2012. Universal Methods of Design: 100 Ways to Research Complex Problems, Develop Innovative Ideas, and Design Effective Solutions. Rockport Publishers. Google-Books-ID: pCVATIpzYfUC.

[27] Eva Hornecker. 2005. A design theme for tangible interaction: embodied facilitation. In ECSCW 2005. Springer, 23-43.

[28] Petra Isenberg, Danyel Fisher, Meredith Ringel Morris, Kori Inkpen, and Mary Czerwinski. 2010. An exploratory study of co-located collaborative visual analytics around a tabletop display. In 2010 IEEE Symposium on Visual Analytics Science and Technology. IEEE, 179-186.

[29] Hiroshi Ishii, Daniel Leithinger, Sean Follmer, Amit Zoran, Philipp Schoessler, and Jared Counts. 2015. TRANSFORM: Embodiment of "Radical Atoms" at Milano Design Week. In Proceedings of the 33rd Annual ACM Conference Extended Abstracts on Human Factors in Computing Systems (CHI EA '15). Association for Computing Machinery, New York, NY, USA, 687-694. DOI : http://dx.doi.org/10.1145/2702613.2702969

[30] Mikkel R. Jakobsen and Kasper Hornbæk. 2014. Up Close and Personal: Collaborative Work on a High-Resolution Multitouch Wall Display. ACM Trans.
Comput.-Hum. Interact. 21, 2, Article Article 11 (Feb. 2014), 34 pages. DOI :

http://dx.doi.org/10.1145/2576099

[31] Sungjune Jang, Lawrence H. Kim, Kesler Tanner, Hiroshi Ishii, and Sean Follmer. 2016. Haptic Edge Display for Mobile Tactile Interaction. In Proceedings of the 2016 CHI Conference on Human Factors in Computing Systems (CHI '16). Association for Computing Machinery, New York, NY, USA, 3706-3716. DOI :

http://dx.doi.org/10.1145/2858036.2858264

[32] Mads Møller Jensen, Sarah-Kristin Thiel, Eve Hoggan, and Susanne Bødker. 2018. Physical Versus Digital Sticky Notes in Collaborative Ideation. Computer Supported Cooperative Work (CSCW) 27, 3-6 (2018), 609-645.

[33] Magnus H. Kaspersen, Sebastian Hines, Mark Moore, Majken K. Rasmussen, and Marcelo A. Dias. 2019. Lifting Kirigami Actuators Up Where They Belong: Possibilities for SCI. In Proceedings of the 2019 on Designing Interactive Systems Conference (DIS '19). Association for Computing Machinery, New York, NY, USA, 935-947. D0I : http://dx.doi.org/10.1145/3322276.3323688

[34] Adam Kendon. 1990. Spatial organization in social encounters: The F-formation system. Conducting interaction: Patterns of behavior in focused encounters (1990).

[35] Finn Kensing and Kim Halskov Madsen. 1992. Design at Work. L. Erlbaum Associates Inc., Hillsdale, NJ, USA, Chapter Generating Visions: Future Workshops and Metaphorical Design, 155-168.

http://dl. acm.org/citation. cfm?id=125470.125433

[36] Sunkook Kim, Hyuk-Jun Kwon, Sunghun Lee, Hongshik Shim, Youngtea Chun, Woong Choi, Jinho Kwack, Dongwon Han, MyoungSeop Song, Sungchul Kim, and others. 2011. Low-power flexible organic light-emitting diode display device. Advanced Materials 23, 31 (2011), 3511-3516.

[37] Clemens N. Klokmose, James R. Eagan, Siemen Baader, Wendy Mackay, and Michel Beaudouin-Lafon. 2015. Webstrates: Shareable Dynamic Media. In Proceedings of the 28th Annual ACM Symposium on User Interface Software \& Technology (UIST'15). Association for Computing Machinery, New York, NY, USA, 280-290. DOI : http://dx.doi .org/10.1145/2807442 .2807446

[38] Peter Gall Krogh, Marianne Graves Petersen, Kenton O’Hara, and Jens Emil Grønbæk. 2017. Sensitizing Concepts for Socio-Spatial Literacy in HCI. In Proceedings of the 2017 CHI Conference on Human Factors in Computing Systems (CHI'17). Association for Computing Machinery, New York, NY, USA, 6449-6460. DOI :

http://dx.doi.org/10.1145/3025453.3025756 
[39] Russell Kruger, Sheelagh Carpendale, Stacey D Scott, and Saul Greenberg. 2004. Roles of orientation in tabletop collaboration: Comprehension, coordination and communication. Computer Supported Cooperative Work (CSCW) 13, 5-6 (2004), 501-537.

[40] Vijay Kumar. 2012. 101 Design Methods: A Structured Approach for Driving Innovation in Your Organization (1 edition ed.). Wiley, Hoboken, N.J.

[41] Sang-Wol Lee and In-Seo Kee. 2014. Foldable display device. (Aug. 12 2014). US Patent 8,804,349.

[42] Daniel Leithinger, Sean Follmer, Alex Olwal, and Hiroshi Ishii. 2015. Shape displays: Spatial interaction with dynamic physical form. IEEE computer graphics and applications 35, 5 (2015), 5-11.

[43] Daniel Leithinger, Sean Follmer, Alex Olwal, Samuel Luescher, Akimitsu Hogge, Jinha Lee, and Hiroshi Ishii. 2013. Sublimate: State-Changing Virtual and Physical Rendering to Augment Interaction with Shape Displays. In Proceedings of the SIGCHI Conference on Human Factors in Computing Systems (CHI'13). Association for Computing Machinery, New York, NY, USA, 1441-1450. DOI :

http://dx.doi.org/10.1145/2470654.2466191

[44] Daniel Leithinger, David Lakatos, Anthony DeVincenzi, Matthew Blackshaw, and Hiroshi Ishii. 2011. Direct and gestural interaction with relief: a $2.5 \mathrm{D}$ shape display. In Proceedings of the 24th annual ACM symposium on User interface software and technology. ACM, 541-548.

[45] David Lindlbauer, Jens Emil Grønbæk, Morten Birk, Kim Halskov, Marc Alexa, and Jörg Müller. 2016. Combining Shape-Changing Interfaces and Spatial Augmented Reality Enables Extended Object Appearance. In Proceedings of the 2016 CHI Conference on Human Factors in Computing Systems (CHI '16). Association for Computing Machinery, New York, NY, USA, 791-802. DOI : http://dx.doi.org/10.1145/2858036.2858457

[46] Paul Marshall, Yvonne Rogers, and Nadia Pantidi. 2011. Using F-Formations to Analyse Spatial Patterns of Interaction in Physical Environments. In Proceedings of the ACM 2011 Conference on Computer Supported Cooperative Work (CSCW'11). Association for Computing Machinery, New York, NY, USA, 445-454. DOI:http://dx.doi.org/10.1145/1958824.1958893

[47] Sven Mayer, Lars Lischke, Jens Emil Grønbæk, Zhanna Sarsenbayeva, Jonas Vogelsang, Paweł W. Woundefinedniak, Niels Henze, and Giulio Jacucci. 2018. Pac-Many: Movement Behavior When Playing Collaborative and Competitive Games on Large Displays. In Proceedings of the 2018 CHI Conference on Human Factors in Computing Systems (CHI '18). Association for Computing Machinery, New York, NY, USA, Article Paper 539, 10 pages. DOI : http://dx.doi .org/10.1145/3173574.3174113

[48] Will McGrath, Brian Bowman, David McCallum, Juan David Hincapié-Ramos, Niklas Elmqvist, and
Pourang Irani. 2012. Branch-Explore-Merge:

Facilitating Real-Time Revision Control in Collaborative Visual Exploration. In Proceedings of the 2012 ACM International Conference on Interactive Tabletops and Surfaces (ITS '12). Association for Computing Machinery, New York, NY, USA, 235-244. DOI : http://dx. doi .org/10.1145/2396636.2396673

[49] Helena M. Mentis. 2017. Collocated Use of Imaging Systems in Coordinated Surgical Practice. Proc. ACM Hum.-Comput. Interact. 1, CSCW, Article 78 (Dec. 2017), 17 pages. DOI :

http://dx.doi.org/10.1145/3134713

[50] Helena M. Mentis, Kenton O'Hara, Abigail Sellen, and Rikin Trivedi. 2012. Interaction Proxemics and Image Use in Neurosurgery. In Proceedings of the SIGCHI Conference on Human Factors in Computing Systems (CHI '12). Association for Computing Machinery, New York, NY, USA, 927-936. DOI :

http://dx.doi.org/10.1145/2207676.2208536

[51] Meredith Ringel Morris, Anthony Cassanego, Andreas Paepcke, Terry Winograd, Ann Marie Piper, and Anqi Huang. 2006a. Mediating group dynamics through tabletop interface design. IEEE computer graphics and applications 26, 5 (2006), 65-73.

[52] Meredith Ringel Morris, Andreas Paepcke, Terry Winograd, and Jeannie Stamberger. 2006b. TeamTag: Exploring Centralized versus Replicated Controls for Co-Located Tabletop Groupware. In Proceedings of the SIGCHI Conference on Human Factors in Computing Systems (CHI '06). Association for Computing Machinery, New York, NY, USA, 1273-1282. DOI : http://dx.doi .org/10.1145/1124772.1124964

[53] Sara Nabil, Aluna Everitt, Miriam Sturdee, Jason Alexander, Simon Bowen, Peter Wright, and David Kirk. 2018. ActuEating: Designing, Studying and Exploring Actuating Decorative Artefacts. In Proceedings of the 2018 Designing Interactive Systems Conference (DIS '18). Association for Computing Machinery, New York, NY, USA, 327-339. DOI :

http://dx.doi.org/10.1145/3196709.3196761

[54] Kenton O'hara, Jesper Kjeldskov, and Jeni Paay. 2011. Blended Interaction Spaces for Distributed Team Collaboration. ACM Trans. Comput.-Hum. Interact. 18, 1, Article Article 3 (May 2011), 28 pages. DOI : http://dx.doi.org/10.1145/1959022.1959025

[55] Kas Oosterhuis and Nimish Biloria. 2008. Interactions with Proactive Architectural Spaces: The Muscle Projects. Commun. ACM 51, 6 (June 2008), 70-78. DOI: http://dx. doi .org/10.1145/1349026.1349041

[56] Peter Peltonen, Esko Kurvinen, Antti Salovaara, Giulio Jacucci, Tommi Ilmonen, John Evans, Antti Oulasvirta, and Petri Saarikko. 2008. It's Mine, Don't Touch! Interactions at a Large Multi-Touch Display in a City Centre. In Proceedings of the SIGCHI Conference on Human Factors in Computing Systems (CHI'08). 
Association for Computing Machinery, New York, NY, USA, 1285-1294. DOI :

http://dx.doi.org/10.1145/1357054.1357255

[57] David Pinelle, Miguel Nacenta, Carl Gutwin, and Tadeusz Stach. 2008. The effects of co-present embodiments on awareness and collaboration in tabletop groupware. In Proceedings of graphics interface 2008. Canadian Information Processing Society, 1-8.

[58] Majken Kirkegård Rasmussen, Timothy Merritt, Miguel Bruns Alonso, and Marianne Graves Petersen. 2016. Balancing User and System Control in Shape-Changing Interfaces: A Designerly Exploration. In Proceedings of the TEI '16: Tenth International Conference on Tangible, Embedded, and Embodied Interaction (TEI'16). Association for Computing Machinery, New York, NY, USA, 202-210. DOI : http://dx.doi.org/10.1145/2839462.2839499

[59] Yvonne Rogers and Siân Lindley. 2004. Collaborating around vertical and horizontal large interactive displays: which way is best? Interacting with Computers 16, 6 (2004), 1133-1152.

[60] Anne Roudaut, Abhijit Karnik, Markus Löchtefeld, and Sriram Subramanian. 2013. Morphees: Toward High "Shape Resolution" in Self-Actuated Flexible Mobile Devices. In Proceedings of the SIGCHI Conference on Human Factors in Computing Systems (CHI'13). Association for Computing Machinery, New York, NY, USA, 593-602. DOI : http://dx.doi.org/10.1145/2470654.2470738

[61] Kathy Ryall, Clifton Forlines, Chia Shen, and Meredith Ringel Morris. 2004. Exploring the Effects of Group Size and Table Size on Interactions with Tabletop Shared-Display Groupware. In Proceedings of the 2004 ACM Conference on Computer Supported Cooperative Work (CSCW'04). Association for Computing Machinery, New York, NY, USA, 284-293. DOI : http://dx.doi.org/10.1145/1031607.1031654

[62] Harpreet Sareen, Udayan Umapathi, Patrick Shin, Yasuaki Kakehi, Jifei Ou, Hiroshi Ishii, and Pattie Maes. 2017. Printflatables: Printing Human-Scale, Functional and Dynamic Inflatable Objects. In Proceedings of the 2017 CHI Conference on Human Factors in Computing Systems (CHI '17). Association for Computing Machinery, New York, NY, USA, 3669-3680. DOI : http://dx.doi.org/10.1145/3025453.3025898

[63] Stacey D. Scott, M. Sheelagh T. Carpendale, and Kori M. Inkpen. 2004. Territoriality in Collaborative Tabletop Workspaces. In Proceedings of the 2004 ACM Conference on Computer Supported Cooperative Work (CSCW '04). Association for Computing Machinery, New York, NY, USA, 294-303. DOI : http://dx.doi.org/10.1145/1031607.1031655

[64] Stacey D Scott, Karen D Grant, and Regan L Mandryk. 2003. System guidelines for co-located, collaborative work on a tabletop display. In ECSCW 2003. Springer, 159-178.
[65] Norbert A. Streitz, Jörg Geiundefinedler, Torsten Holmer, Shin'ichi Konomi, Christian Müller-Tomfelde, Wolfgang Reischl, Petra Rexroth, Peter Seitz, and Ralf Steinmetz. 1999. I-LAND: An Interactive Landscape for Creativity and Innovation. In Proceedings of the SIGCHI Conference on Human Factors in Computing Systems (CHI '99). Association for Computing Machinery, New York, NY, USA, 120-127. DOI : http://dx.doi.org/10.1145/302979.303010

[66] Kazuki Takashima, Naohiro Aida, Hitomi Yokoyama, and Yoshifumi Kitamura. 2013. TransformTable: A Self-Actuated Shape-Changing Digital Table. In Proceedings of the 2013 ACM International Conference on Interactive Tabletops and Surfaces (ITS '13). Association for Computing Machinery, New York, NY, USA, 179-188. DOI :

http://dx.doi.org/10.1145/2512349.2512818

[67] Kazuki Takashima, Takafumi Oyama, Yusuke Asari, Ehud Sharlin, Saul Greenberg, and Yoshifumi Kitamura. 2016. Study and Design of a Shape-Shifting Wall Display. In Proceedings of the 2016 ACM Conference on Designing Interactive Systems (DIS '16). Association for Computing Machinery, New York, NY, USA, 796-806. DOI : http://dx.doi.org/10.1145/2901790.2901892

[68] Peter Tandler, Thorsten Prante, Christian Müller-Tomfelde, Norbert Streitz, and Ralf Steinmetz. 2001. Connectables: Dynamic Coupling of Displays for the Flexible Creation of Shared Workspaces. In Proceedings of the 14th Annual ACM Symposium on User Interface Software and Technology (UIST'01). Association for Computing Machinery, New York, NY, USA, 11-20. DOI :

http://dx.doi.org/10.1145/502348.502351

[69] Anthony Tang, Melanie Tory, Barry Po, Petra Neumann, and Sheelagh Carpendale. 2006. Collaborative Coupling over Tabletop Displays. In Proceedings of the SIGCHI Conference on Human Factors in Computing Systems (CHI '06). Association for Computing Machinery, New York, NY, USA, 1181-1190. DOI : http://dx.doi.org/10.1145/1124772.1124950

[70] Martin Tomitsch, Cara Wrigley, Madeleine Borthwick, Ahmadpour Naseem, Jessica Frawley, Baki Kocaballi, Claudia Nunez-Pacheco, Karla Straker, and Lian Loke. 2018. Design. Think. Make. Break. Repeat. A Handbook of Methods. BIS Publishers.

[71] Lili Tong, Audrey Serna, Simon Pageaud, Sébastien George, and Aurélien Tabard. 2016. It's Not How You Stand, It's How You Move: F-Formations and Collaboration Dynamics in a Mobile Learning Game. In Proceedings of the 18th International Conference on Human-Computer Interaction with Mobile Devices and Services (MobileHCI '16). Association for Computing Machinery, New York, NY, USA, 318-329. DOI : http://dx.doi.org/10.1145/2935334.2935343 
[72] Anke van Oosterhout, Miguel Bruns Alonso, and Satu Jumisko-Pyykkö. 2018. Ripple Thermostat: Affecting the Emotional Experience through Interactive Force Feedback and Shape Change. In Proceedings of the 2018 CHI Conference on Human Factors in Computing Systems (CHI'18). Association for Computing Machinery, New York, NY, USA, Article Paper 655, 12 pages. DOI : http://dx.doi.org/10.1145/3173574.3174229

[73] James R. Wallace, Nancy Iskander, and Edward Lank. 2016. Creating Your Bubble: Personal Space On and Around Large Public Displays. In Proceedings of the 2016 CHI Conference on Human Factors in Computing Systems (CHI'16). Association for Computing Machinery, New York, NY, USA, 2087-2092. DOI : http://dx.doi.org/10.1145/2858036.2858118

[74] Malte Weiss, Simon Voelker, Christine Sutter, and Jan Borchers. 2010. BendDesk: Dragging across the Curve. In ACM International Conference on Interactive Tabletops and Surfaces (ITS '10). Association for Computing Machinery, New York, NY, USA, 1-10. DOI : http://dx.doi.org/10.1145/1936652.1936654

[75] Andrew D. Wilson and Hrvoje Benko. 2010. Combining Multiple Depth Cameras and Projectors for Interactions on, above and between Surfaces. In Proceedings of the 23nd Annual ACM Symposium on User Interface Software and Technology (UIST'10). Association for Computing Machinery, New York, NY, USA, 273-282. DOI : http://dx.doi .org/10.1145/1866029.1866073

[76] Lining Yao, Ryuma Niiyama, Jifei Ou, Sean Follmer, Clark Della Silva, and Hiroshi Ishii. 2013. PneUI: Pneumatically Actuated Soft Composite Materials for Shape Changing Interfaces. In Proceedings of the 26th Annual ACM Symposium on User Interface Software and Technology (UIST '13). Association for Computing Machinery, New York, NY, USA, 13-22. DOI : http://dx.doi.org/10.1145/2501988.2502037

[77] Johannes Zagermann, Ulrike Pfeil, Roman Rädle, Hans-Christian Jetter, Clemens Klokmose, and Harald Reiterer. 2016. When Tablets Meet Tabletops: The Effect of Tabletop Size on Around-the-Table Collaboration with Personal Tablets. In Proceedings of the 2016 CHI Conference on Human Factors in Computing Systems (CHI '16). Association for Computing Machinery, New York, NY, USA, 5470-5481. DOI :

http://dx.doi.org/10.1145/2858036.2858224 